\title{
Pathophysiology of unilateral asterixis due to thalamic lesion.
}

\section{AUTHOR(S):}

Inoue, Manabu; Kojima, Yasuhiro; Mima, Tatsuya;

Sawamoto, Nobukatsu; Matsuhashi, Masao; Fumuro,

Tomoyuki; Kinboshi, Masato; Koganemaru, Satoko; Kanda, Masutarou; Shibasaki, Hiroshi

\section{CITATION:}

Inoue, Manabu ... [et al]. Pathophysiology of unilateral asterixis due to thalamic lesion.. Clinical neurophysiology : official journal of the International Federation of Clinical Neurophysiology 2012, 123(9): 1858-1864

\section{ISSUE DATE:}

2012-09

URL:

http://hdl.handle.net/2433/160142

\section{RIGHT:}

(c) 2012 International Federation of Clinical Neurophysiology. Published by Elsevier Ireland Ltd.; This is not the published version. Please cite only the published version.; この 論文は出版社版でありません。引用の際には出版社版をご確認ご利用ください。 
Pathophysiology of unilateral asterixis due to thalamic lesion

Manabu Inoue, ${ }^{1,2}$ Yasuhiro Kojima, ${ }^{1}$ Tatsuya Mima, ${ }^{2}$ Nobukatsu Sawamoto, ${ }^{2}$ Masao Matsuhashi, 2 Tomoyuki Fumuro, ${ }^{3}$ Masato Kinboshi, ${ }^{1}$ Satoko Koganemaru, ${ }^{2}$ Masutarou Kanda ${ }^{1}$ and Hiroshi Shibasaki ${ }^{4}$

Author Affiliations

${ }^{1}$ Department of Neurology, Ijinkai Takeda General Hospital, Kyoto 601-1495, Japan

${ }^{2}$ Human Brain Research Center, Kyoto University Graduate School of Medicine, Kyoto,606-8507 Japan

${ }^{3}$ Department of Neurology, Kyoto University Graduate School of Medicine, Kyoto 606-8507, Japan

${ }^{4}$ Emeritus Professor, Kyoto University Graduate School of Medicine, Kyoto 606-8507, Japan

Correspondence to: Manabu Inoue, M.D., Department of Neurology, Ijinkai Takeda General Hospital, Fushimi-ku, Kyoto city, Kyoto 601-1495, Japan.

Tel: +81-75-572-6331; Fax: +81-75-571-8877

E-mail: gaku@kuhp.kyoto-u.ac.jp

Footnote:

The cases were presented at ICCN2010 (Kobe) in Japan. 


\section{Highlights}

Two cases with unilateral asterixis caused by an infarction in the lateral thalamus were studied by using electrophysiological and neuroimaging methods.

Averaging of EEG time-locked to the asterixis revealed rhythmic oscillations of beta band at the central area contralateral to the affected hand.

The present study for the first time demonstrated that the asterixis due to thalamic lesion is mediated by the sensorimotor cortex which is rendered in an excessive inhibition. 


\section{Abstract}

Objective: Unilateral asterixis has been reported in patients with thalamic lesion. This study is aimed at elucidating pathophysiology of the thalamic asterixis.

Methods: Two cases with unilateral asterixis caused by an infarction in the lateral thalamus were studied by using analysis of the asterixis-related cortical activities, transcranial magnetic stimulation (TMS) for motor cortex excitability, and probabilistic diffusion tractography for the thalamo-cortical connectivity.

Results: Averaging of EEG time-locked to the asterixis revealed rhythmic oscillations of beta band at the central area contralateral to the affected hand. TMS revealed a decrease of motor evoked potential (MEP) amplitude and a prolongation of silent period (SP). The anatomical mapping of connections between the thalamus and cortical areas using diffusion weighted image (DWI) showed that the lateral thalamus involved by the infarction was connected to the premotor cortex, the primary motor cortex (M1) and the primary somatosensory cortex (S1) of the corresponding hemisphere.

Conclusions: The thalamic asterixis is mediated by the sensorimotor cortex, which is rendered in an excessive inhibition as a result of the thalamic lesion involving the ventral lateral nucleus.

Significance: This is the first demonstration of participation of the 
sensorimotor cortex in the generation of asterixis due to the lateral thalamic lesion.

Key Words: thalamic asterixis; thalamic infarction; asterixis-related cortical activity; motor cortex excitability; thalamo-cortical connectivity 


\section{Introduction}

Asterixis, a lapse in sustained posture due to intermittent interruption of muscle contraction, was originally described by Adams and Foley in patients with hepatic encephalopathy (Adams and Foley, 1953). Later, asterixis has been reported in a variety of conditions including metabolic or toxic encephalopathy and focal central nervous system (CNS) lesion (Degos et al., 1979, Calzetti et al., 1983, Young and Shahani, 1986, Rio et al., 1995). In patients with encephalopathy, asterixis commonly involves bilateral limb muscles, whereas asterixis caused by focal CNS lesions is focal or unilateral. Unilateral asterixis has been reported in patients with thalamic lesion, especially the lesion involving the lateral thalamus (Donat, 1980, Tatu et al., 2000, Klos and Wijdicks, 2006).

In patients with encephalopathy, two types of surface electromyographic (EMG) pattern of asterixis were demonstrated; pure silent period (SP) (type I) and SP following a brief EMG discharge (type II) (Ugawa et al., 1989). The surface EMG patterns of asterixis caused by thalamic lesion are similar to those seen in encephalopathy except for the different distribution (Tatu et al., 2000). Therefore, the pathophysiology of asterixis caused by a focal CNS lesion is important for elucidating the mechanism of asterixis caused by other etiologies. However, the pathophysiology of thalamic asterixis has not been clearly 
understood.

In the present study on two cases with thalamic infarction manifesting asterixis in the contralateral hand, we investigated a possible participation of the cerebral cortex in the generation of asterixis by using electrophysiological and neuroimaging methods. Cortical activities associated with asterixis were analyzed by the SP-locked back averaging of electroencephalogram (EEG) (Ugawa et al., 1989, Ugawa et al., 1990, Shibasaki, 1995) and by an analysis of the power change of rhythmic EEG oscillations time-locked to the asterixis (Ohara et al., 2000, Ohara et al., 2001). Furthermore, excitability of the primary motor cortex (M1) was studied by trancranial magnetic stimulation (TMS) (Rothwell, 1999, Begum et al., 2005). Anatomically, the connectivity of the thalamic subregions involved by infarction to cortical areas was investigated by the probabilistic diffusion tractography (Behrens et al., 2003).

The present study for the first time demonstrated that the asterixis due to thalamic lesion is mediated by the sensorimotor cortex which is rendered in an excessive inhibition as a result of the thalamic lesion. Anatomically, the thalamic subregions involved by infarction were shown to be connected to the premotor cortex, the primary motor cortex (M1) and the primary somatosensory cortex (S1). 


\section{Patients}

\section{Case 1}

A 71-year-old man was admitted because of loss of balance, numbness in the right hand, and difficulty in grabbing a door knob with the hand. The symptoms occurred suddenly and worsened in their degree over the following several hours. His past medical history included hypertension and diabetes mellitus with $\mathrm{HbA}_{1} \mathrm{c}$ of $10 \%$. On general physical examination, blood pressure was $171 / 75 \mathrm{mmHg}$, and pulse rate was $80 / \mathrm{min}$ and regular. There was no cardiac murmur or vascular bruit in the neck. On neurological examination, orientation and recent memory were intact. There was no abnormality in eye movements except for minimal horizontal gaze nystagmus to both directions. Speech was not dysarthric. Muscle tonus, strength and deep tendon reflexes were normal and symmetric. Incoordination and dysmetria were moderately seen in the right upper limb and markedly in the right lower limb. Asterixis and occasional myoclonic jerks were seen in the right hand. Asterixis was recognized only while the right wrist was kept in extended position. Neither myoclonic jerk nor asterixis was sensitive to any external stimulus. The right leg was unsteady especially on turning, but tandem standing was relatively good. Plantar reflex was flexor on the left and non-flexor on the right, but Babinski or Chaddock sign was negative. Tactile sensation 
was mildly decreased with dysesthesia in the right hand, but there was no other sensory deficit. There was no pseudoathetosis.

Fasting blood sugar was $261 \mathrm{mg} / \mathrm{dl}$, and total cholesterol was $243 \mathrm{mg} / \mathrm{dl}$, but there were no other metabolic abnormalities. The conventional magnetic resonance image (MRI) was obtained by a 1.5 Tesla whole-body MR scanner (Vantage; Toshiba Medical Systems, Tochigi, Japan) on the day of admission. Diffusion weighted image (DWI) was obtained using echo planar imaging (EPI) covering the whole brain in an axial orientation ( $\mathrm{TR}=6,500$ $\mathrm{ms}, \mathrm{TE}=120 \mathrm{~ms}, \mathrm{FA}=90^{\circ}$, slice thickness $=6 \mathrm{~mm}$, slice gap $=1.2$ $\mathrm{mm}, \mathrm{FOV}=200 \times 200 \mathrm{~mm}$, matrix size $=144 \times 96,20$ slices $). \quad$ On the DWI, an isolated, high signal intensity lesion suggestive of a new infarction was seen in the lateral part of the left thalamus (Fig. 1). Electrophysiologically, the nerve conduction study showed slightly prolonged distal latency in the median nerve and slightly slow conduction velocity in the median, tibial and sural nerves, which suggested polyneuropathy. Somatosensory evoked potential (SEP) following the right median nerve stimulation was barely recognizable while the onset-peak amplitude of N20 following the left median nerve stimulation was $0.34 \mu \mathrm{V}$. The peak latency of N20 of the left median nerve SEP was slightly delayed to $22.1 \mathrm{~ms}$, most likely due to diabetic neuropathy. There was no giant SEP. 
Case 2

A 69-year-old man was admitted because of sudden onset of numbness and tingling sensation on the right face and the right upper and lower limbs, followed by unsteadiness with tendency to fall to the right on the following day. The patient felt the right leg warmer than the left. He had a history of diabetes mellitus, but no hypertension. Right parotid tumor was operated 3 years before the admission, resulting in facial paresis, which subsequently improved. He was a heavy smoker until two years prior to the admission. On general physical examination, blood pressure was $156 / 80 \mathrm{mmHg}$, and pulse rate was 60/min and regular. A surgical scar was seen in the right mandibular skin. There was no cardiac murmur or vascular bruit in the neck. On neurological examination, orientation and recent memory were intact. Speech was slightly dysarthric. There was mild horizontal nystagmus to the right on lateral gaze to the right. Muscle tonus and strength were normal and symmetric. Ataxia was seen moderately in the right hand and mildly in the right leg. Occasionally asterixis was seen in the right hand. The asterixis was not sensitive to any external stimulus. Standing was unstable with slight truncal deviation to the right. Gait was also unstable with tendency to fall to the right. Tandem gait was impossible without support. Deep tendon reflexes were normal 
and symmetric, but the right plantar reflex was extensor. Pain, temperature and tactile sensations were moderately decreased on the right side of the body including the face. Vibration sense and joint position sense were moderately diminished in the right hand. There was no hyperpathia. The laboratory data showed diabetes mellitus with fasting blood sugar of $290 \mathrm{mg} / \mathrm{dl}$ and $\mathrm{HbA} 1 \mathrm{C}$ of $8.4 \%$, but there were no other metabolic abnormalities. Electrophysiologically, the nerve conduction study was normal. In the median nerve SEP, the onset-peak amplitude of N20 was lower on the right $(0.16 \mu \mathrm{V})$ than on the left $(1.83 \mu \mathrm{V})$, although the peak latency of N20 was normal $(19.6 \mathrm{~ms}$ on the right and $19.3 \mathrm{~ms}$ on the left). There was no giant SEP. On the conventional DWI, a high signal intensity lesion was found in the lateral part of the left thalamus (Fig. 1). There were no other abnormalities on the MRI.

\section{Methods}

Analysis of cortical activities time-locked to asterixis

EEG and EMG were simultaneously recorded 10 days (Case 1) and 8 days (Case 2) after the clinical onset, when asterixis occurred frequently in both cases. All EEG electrodes were placed over the scalp according to the 10-20 International Electrode System (Klem et al., 1999). The EMGs were recorded from the extensor carpi radialis (ECR) and flexor carpi ulnaris 
(FCU) muscles with a pair of cup electrodes placed $3 \mathrm{~cm}$ apart on the skin overlying each muscle. The signals were recorded with a bandpass filter of $0.016-300 \mathrm{~Hz}$ for EEG and EMG, and digitized at a sampling rate of $1000 \mathrm{~Hz}$ and stored on the magneto-optical disks equipped with EEG machine (Neurofax, Nihon Kohden, Tokyo, Japan). SP-locked averaging of EEG was carried out off-line(Ugawa et al., 1989). As for the SP onset, the onset of EMG interruption was adopted for type $1 \mathrm{SP}$, and the onset of the second EMG interruption was adopted for type 2 SP. Although the number of sweeps for averaging was limited because of infrequent occurrence of asterixis at the time of the recording, more than two sets of data were averaged for each case. After confirming the reproducibility of the average data, the group average was obtained for each case.

Power change of cortical oscillations time-locked to asterixis

The frequency domain analysis was done on the same EEG data as used for the time domain analysis (see above). The asterixis-related power change of EEG oscillations recorded from 9 scalp electrodes (C1, C2, FC3, FC4, C3, C4, CP3, CP4, Cz) was calculated using a fast Fourier transform algorithm implemented on Matlab 7.6 (Mathworks, Natick, MA) and log-transformed to stabilize the variance. To obtain the time course of power, the center of the moving window was shifted from $0.5 \mathrm{~s}$ before to $0.5 \mathrm{~s}$ 
after the SP onset in steps of $0.05 \mathrm{~s}$. The baseline period was defined as the segment from $0.5 \mathrm{~s}$ before the SP onset to the SP onset. The frequency bands up to $100 \mathrm{~Hz}$ in steps of $5 \mathrm{~Hz}$ were analyzed, and the time-frequency maps of power change were constructed.

\section{Analysis of cortical excitability}

To investigate the M1 excitability, TMS was applied with a figure-of-eight shaped coil (outer diameter of each coil: $9 \mathrm{~cm}$ ) connected to the Magstim 200 stimulator through the Bistim module (Magstim Co., Whitland, UK). The test was performed 35 days (Case 1) and 45 days (Case 2) after the clinical onset when asterixis occurred infrequently. EMGs were bipolarly recorded from the right and left first dorsal interosseous (FDI) muscles using silver electrodes. Stimulus intensity was expressed as a percentage of the maximum stimulator output. The coil was placed tangentially to the scalp and held approximately 45 degrees to the mid-sagittal line at the optimal position for eliciting the maximum motor evoked potential (MEP) in the target FDI. Resting motor threshold (rMT) and active motor threshold (aMT) were determined in a conventional way (Rossini et al., 1994).

To evaluate the excitability of M1, the stimulus intensity was set to the intensity that produced MEP amplitude of 
approximately $1 \mathrm{mV}$ in the right FDI for each case. That was $70 \%$ and $75 \%$ of the maximal machine output in Case 1 and Case 2 , respectively. To investigate the inhibitory function of $\mathrm{M} 1$, the duration of the TMS-induced SP and the short-interval intracortical inhibition (SICI) were measured (Rothwell, 1999). The SP was elicited by a single pulse TMS with an intensity of $140 \%$ of rMT during $40 \%$ voluntary contraction of the target muscle and measured from the TMS onset. The SICI was measured using double pulse TMS combining a sub-threshold conditioning pulse (95\% of aMT) and the supra-threshold test-pulse with a $3 \mathrm{~ms}$ interval. The SICI was calculated as the ratio of the paired MEP divided by the test-alone MEP.

\section{Neuroimaging study for thalamo-cortical connectivity}

Images for probabilistic diffusion tractography were acquired with a 3 Tesla whole-body MR scanner (Trio; Siemens, Erlangen, Germany) on the same day as for the TMS study. The cortico-thalamic connectivity was calculated on each hemisphere including the lesioned sites of the thalamus. However, the data related to the lesioned sites were interpreted by taking into account the data obtained from the corresponding sites of the intact hemisphere. DWI was performed using EPI covering the whole brain in an axial orientation $(\mathrm{TR}=10,500 \mathrm{~ms}, \mathrm{TE}=96 \mathrm{~ms}$, $\mathrm{FA}=90^{\circ}$, voxel size $=2 \times 2 \times 2 \mathrm{~mm}, \mathrm{FOV}=192 \times 192 \mathrm{~mm}$, matrix 
size $=96 \times 96,70$ slices). The diffusion weighting was isotropically distributed along 81 directions by using a b value of $1,500 \mathrm{~s} / \mathrm{mm}^{2}$. Nine volumes with non-diffusion weighting $(b=0$ $\mathrm{s} / \mathrm{mm}^{2}$ ) were also acquired at points throughout the data acquisition. A T1 weighted anatomical image of the whole brain was acquired using a magnetization prepared rapid gradient echo sequence $\left(\mathrm{TR}=2,000 \mathrm{~ms}, \mathrm{TE}=4.38 \mathrm{~ms}, \mathrm{FA}=8^{\circ}\right.$, voxel size $=1 \times 1$ $\times 1 \mathrm{~mm}, \mathrm{FOV}=176 \times 192 \mathrm{~mm}$, matrix size $=176 \times 192,160$ slices $)$. Field map data containing phase and magnitude images were obtained by using gradient echo sequences $(\mathrm{TR}=511 \mathrm{~ms}, \mathrm{TE}=$ $7.65 \mathrm{~ms}, \mathrm{FA}=60^{\circ}$, voxel size $=3 \times 3 \times 3 \mathrm{~mm}, \mathrm{FOV}=192 \times 192 \mathrm{~mm}$, matrix size $=64 \times 64,46$ slices for phase images, and $\mathrm{TR}=511 \mathrm{~ms}$, $\mathrm{TE}=5.19 \mathrm{~ms}, \mathrm{FA}=60^{\circ}$, voxel size $=3 \times 3 \times 3 \mathrm{~mm}, \mathrm{FOV}=192 \times 192$ $\mathrm{mm}$, matrix size $=64 \times 64,46$ slices for magnitude images).

Probabilistic diffusion tractography was performed in accordance with the previously reported method (Behrens et al., 2003). In brief, DWI data were analyzed by using the programs contained in FMRIB Software Library (FMRIB Centre, Oxford, UK). After unwarping based on the field map data, DWI data were corrected for eddy currents and head motion by using affine registration to the first $b=0$ reference volume. The local diffusion directions were computed by using Bedpostx. Seven cortical regions for each hemisphere were defined on the atlas as previously reported; prefrontal cortex, premotor cortex, M1, S1, ; 
posterior parietal cortex, occipital cortex, and temporal cortex (Behrens et al., 2003). Thalamus and the seven cortical regions on the atlas were spatially paired for each case using parameters obtained by non-rigid transforming the MR images of atlas to MR images of each case. The probability of connection from each seed voxel in the thalamus to each cortical mask was calculated. An index was assigned to each seed voxel corresponding to the target cortical region with the highest connection probability to the seed. Results were superimposed on the T1 weighted image of each case.

\section{Results}

\section{EMG findings of asterixis}

The surface EMG revealed interruption of sustained muscle contraction corresponding to asterixis in the right ECR and FCU in both cases. Two types of SP were seen in both cases. Type 1 SP was a simple interruption of the ongoing EMG discharge whereas type $2 \mathrm{SP}$ was interrupted by a brief EMG discharge in the middle, producing double SPs (Fig. 2). The duration of the type $1 \mathrm{SP}$ ranged from 42 to $94 \mathrm{~ms}$ in Case 1 and from 31 to $55 \mathrm{~ms}$ in Case 2. The total duration of type 2 SP ranged from 96 to 203 $\mathrm{ms}$ in Case 1 and from 80 to $145 \mathrm{~ms}$ in Case 2. In type $2 \mathrm{SP}$, the first segment was shorter (15-28 ms in Case 1 and $10-28 \mathrm{~ms}$ in 
Case 2) than the second segment (45-139 ms in Case 1 and 35-91 $\mathrm{ms}$ in Case 2). The duration of the sharp EMG transient seen in the middle of type $2 \mathrm{SP}$ was short (30-68 ms in Case 1 and 20-45 $\mathrm{ms}$ in Case 2).

\section{Cortical activities associated with asterixis}

SP-locked average of EEG showed rhythmic activities in the left central area with a main positive peak coinciding with the SP onset in both cases (Fig. 3). SP-locked power change of EEG oscillations showed an increase in the power of beta band in both cases and also at around $80 \mathrm{~Hz}$ in Case 1 in the left central area, starting about $50 \mathrm{~ms}$ before the SP onset (Fig. 4).

\section{Cortical excitability}

On the affected side (left M1), the rMT and aMT were decreased compared with the unaffected side in Case 1 (rMT/aMT: left $42 / 36 \%$, right 47/39\%), whereas both were increased in Case 2 (left 51/40 \%, right 39/31\%). The onset latencies of MEP were the same for both sides in Case 1 (left $21 \mathrm{~ms}$, right $21 \mathrm{~ms}$ ) and nearly the same in Case 2 (left $21.5 \mathrm{~ms}$, right $22 \mathrm{~ms}$ ). SICI on the affected (left) side was lost in Case 1 (left 0.93, right 0.44), whereas it was slightly increased in Case 2 (left 0.33, right 0.57).

Common findings in the two cases were decrease in the MEP amplitude and longer duration of the TMS-induced SP on the 
affected (left) side. The MEP amplitude was smaller on the affected side by approximately $50 \%$ or even more (Case 1: left $1.33 \mathrm{mV}$, right $2.63 \mathrm{mV}$, Case 2: left $1.08 \mathrm{mV}$, right $2.18 \mathrm{mV}$ ) (Fig. 5 ), and the duration of SP on the affected side was longer compared to the unaffected side (Case 1: left $203 \mathrm{~ms}$, right $187 \mathrm{~ms}$, Case 2: left $221 \mathrm{~ms}$, right $189 \mathrm{~ms})$.

\section{Connectivity of subregions of thalamus to cortical areas}

Based on the highest connection probability to each of the seven cortical regions as defined in the Methods, the thalamus was shown to be divided into seven subregions (Fig. 6). The ventrolateral region of the thalamus (light blue) was shown to be connected to M1, the next lateral region (purple) to the premotor cortex, the intermediate region (yellow) to the prefrontal cortex, the next intermediate area (green) to $\mathrm{S} 1$, the area shown in brown to the posterior parietal cortex, the area shown in blue to the occipital cortex, and the dorsal area (pink) to the temporal cortex. The thalamic infarction detected on the T1 weighted image (Fig. 1) involved the lateral areas shown in light blue, purple and green as indicated by a crossbar in Fig. 6. These results suggested that the lesioned thalamic parts were connected to the premotor cortex, M1 and S1 in both cases.

\section{Discussion}


In the two cases investigated in this study, unilateral asterixis acutely developed together with sensory symptoms and ataxia on the same side. Taken together with the findings of MR images, which showed a lesion suggestive of a new infarction in the lateral part of the contralateral thalamus, it is reasonable to ascribe the asterixis to the thalamic lesion. Within the thalamus, the presence of sensory deficits suggested involvement of the ventral posterolateral (VPL) nucleus in Case 1 and VPL as well as ventral posteromedial (VPM) nucleus in Case 2, and ataxia was most likely due to involvement of the ventral lateral (VL) nucleus inrerrupting the cerebellar input. The MRI findings supported this clinical interpretation.

Electrophysiologically, two EMG patterns of asterixis were observed in both cases. Type 1 silent period (SP) was formed of a single period of EMG silence while type $2 \mathrm{SP}$ was interrupted by a brief EMG discharge forming double SPs (Fig. 2). These patterns are similar to those of asterixis seen in epileptic negative myoclonus or cortical negative myoclonus (Ugawa et al., 1989). In these epileptic conditions, the $\mathrm{SP}$ is preceded by a cortical spike arising from the corresponding central area (Ugawa et al., 1989, Guerrini et al., 1993, Tassinari et al., 1998, Shibasaki, 2002) and is often elicited by sensory stimuli, causing cortical reflex negative myoclonus (Shibasaki et al., 1994). Posthypoxic myclonus is also formed by an EMG transient and SP, which is 
associated with an EEG spike and slow wave, respectively (Lance and Adams, 1963). By contrast, the asterixis observed in the present two cases was not associated with a cortical spike, but it was preceded by rhythmic EEG oscillations in the contralateral central area, which corresponds to the hand area of the sensorimotor cortex (Fig. 3). This finding was supported by a power increase of EEG beta band time-locked to the asterixis (Fig. 4). These findings suggest that the thalamic asterixis in the present cases is mediated by the corresponding part of the sensorimotor cortex, but its underlying pathophysiology is different from that of epileptic negative myoclonus.

The power increase of alpha or beta bands time-locked to an event, event-related synchronization (ERS), is considered to be associated with decreased activation of the corresponding cortical area or return to the resting state from the activated condition while the power decrease, event-related desynchronization (ERD), represents increased activation of the cortical area (Pfurtscheller and Aranibar, 1977, Pfurtscheller et al., 2003, Pfurtscheller, 2006, Oishi et al., 2007). Therefore, the present findings suggest that the sensorimotor cortex is in an excessive inhibition associated with each lapse of sustained hand posture.

The above interpretation was further supported by the TMS study. In both cases reported here, the motor evoked potential (MEP) amplitude was decreased and the TMS-induced SP was 
prolonged, suggesting an increase in cortical inhibitory mechanism. As for the motor threshold (MT) and the short-interval intracortical inhibition (SICI), however, there was divergence in results between the two cases. These differences might be related to subtle difference either in the clinical manifestation or in the involved subregions of thalamus between the two cases.

Liepert et al. (2005) studied 8 patients with thalamic infarction electrophysiologically (Liepert et al., 2005). All of their patients showed a decrease in pain, tactile and vibration sense, and mild motor deficits but no asterixis or other involuntary movements. In their cases, MT and maximal MEP amplitude were equal on the two sides, but SICI was decreased and SP was prolonged in the affected hand as compared with the unaffected hand.

In our cases, the MEP amplitude was decreased on the affected side without any overt motor weakness. Thus, the decrease of MEP amplitude might be related to the subclinical suppression of M1 function, which would produce the unilateral transient asterixis. However, further accumulation of similar cases is necessary to confirm this hypothesis.

Regarding a question as to which subregions in the thalamus are responsible for asterixis, the previous reports suggested involvement of the lateral part (Stell et al., 1994, Tatu et al., 2000). The present study using diffusion imaging for the first 
time showed the anatomical connections between the cortical areas and the damaged sites of the thalamus in patients with thalamic asterixis. The thalamic subregion involved by infarction was shown to be connected to the premotor cortex, the primary motor cortex (M1) and primary somatosensory cortex (S1), and this thalamic region contained the VL, ventral anterior, lateral posterior, and VPL nucleus. Among the thalamic nuclei, the small lesions caused by VL thalamotomies were reported to produce asterixis (Young et al., 1976). Oscillations of beta band were postulated to originate from the cerebellum, which leads to increased corticospinal coherence and abnormal expression of the drive at the spinal level (Brown, 2007). Therefore, the VL nucleus that was shown to be involved by infarction in the present cases might be responsible for the increased cortical oscillations and asterixis. However, the mechanism as to how interruption of the cerebellar input by the VL lesion enhanced beta oscillations at the sensorimotor cortex in the present cases remains unsolved.

Beta oscillations at the sensorimotor cortex were reported in a patient with hepatic encephalopathy who presented with asterixis (negative myoclonus) (Ugawa et al., 2003). They reported similar oscillations also in patients with positive cortical myoclonus. Therefore, the beta oscillations may not be specific for the negative motor phenomena like asterixis. Alternatively, abnormal synchrony of the beta band might represent an 
enhanced state of cortical drive to the spinal cord, regardless of whether the myoclonus is positive or negative (Ugawa et al., 2003; Brown, 2007).

Based on the present findings, it is postulated that the involved thalamic nuclei, especially the VL nucleus, might cause an abnormally synchronized activity of beta band in the sensorimotor cortex and cause an exaggerated state of cortical inhibition, thus causing asterixis. 


\section{References}

Adams RD, Foley JM. The neurological disorder associated with liver disease. Res Publ Assoc Res Nerv Ment Dis. 1953; 32:198-237

Begum T, Mima T, Oga T, Hara H, Satow T, Ikeda A, et al. Cortical mechanisms of unilateral voluntary motor inhibition in humans. Neurosci Res. 2005; 53:428-435

Behrens TE, Johansen-Berg H, Woolrich MW, Smith SM, Wheeler-Kingshott CA, Boulby PA, et al. Non-invasive mapping of connections between human thalamus and cortex using diffusion imaging. Nat Neurosci 2003; 6:750-757

Brown P. Abnormal oscillatory synchronisation in the motor system leads to impaired movement. Curr Opin Neurobiol. 2007; 17:656-664

Calzetti S, Gemignani F, Salati MR, Terzano MG. Unilateral asterixis due to thalamic tumor. Case report. Ital J Neurol Sci. 1983; 4:87-90

Degos JD, Verroust J, Bouchareine A, Serdaru M, Barbizet J. Asterixis in focal brain lesions. Arch Neurol. 1979; 36:705-707

Donat JR. Unilateral asterixis due to thalamic hemorrhage. Neurology. 1980; 30:83-84

Guerrini R, Dravet C, Genton P, Bureau M, Roger J, Rubboli G, et al. Epileptic negative myoclonus. Neurology. 1993; 43:1078-1083

Klem GH, Luders HO, Jasper HH, Elger C. The ten-twenty electrode system of the International Federation of Clinical Neurophysiology. Electroenceph Clin Neurophysiol 1999; Suppl 52:3-6

Klos KJ, Wijdicks EF. Unilateral asterixis after thalamic hemorrhage. Neurology. 2006; 66:E11

Lance JW, Adams RD. The syndrome of intention or action myoclonus as a sequel to hypoxic encephalopathy. Brain. 1963; 86:111-136

Liepert J, Restemeyer C, Munchau A, Weiller C. Motor cortex 
excitability after thalamic infarction. Clin Neurophysiol. $2005 ; 116: 1621-1627$

Ohara S, Ikeda A, Kunieda T, Yazawa S, Baba K, Nagamine T, et al. Movement-related change of electrocorticographic activity in human supplementary motor area proper. Brain. 2000; $123: 1203-1215$

Ohara S, Mima T, Baba K, Ikeda A, Kunieda T, Matsumoto R, et al. Increased synchronization of cortical oscillatory activities between human supplementary motor and primary sensorimotor areas during voluntary movements. J Neurosci. 2001; 21:9377-9386

Oishi N, Mima T, Ishii K, Bushara KO, Hiraoka T, Ueki Y, et al. Neural correlates of regional EEG power change.

Neuroimage. 2007; 36:1301-1312

Pfurtscheller G, Aranibar A. Event-related cortical desynchronization detected by power measurements of scalp EEG. Electroencephalogr Clin Neurophysiol. 1977; 42:817-826

Pfurtscheller G, Neuper C, Muller GR, Obermaier B, Krausz G, Schlogl A, et al. Graz-BCI: state of the art and clinical applications. IEEE Trans Neural Syst Rehabil Eng. 2003; $11: 177-180$

Pfurtscheller G. The cortical activation model (CAM). Prog Brain Res. 2006; 159:19-27

Rio J, Montalban J, Pujadas F, Alvarez-Sabin J, Rovira A, Codina A. Asterixis associated with anatomic cerebral lesions: a study of 45 cases. Acta Neurol Scand 1995; 91:377-381 Rossini PM, Barker AT, Berardelli A, Caramia MD, Caruso G, Cracco RQ, et al. Non-invasive electrical and magnetic stimulation of the brain, spinal cord and roots: basic principles and procedures for routine clinical application. Report of an IFCN committee. Electroencephalogr Clin Neurophysiol. 1994; 91:79-92

Rothwell JC. Paired-pulse investigations of short-latency intracortical facilitation using TMS in humans. Electroencephalogr Clin Neurophysiol. 1999; Suppl 
51:113-119

Shibasaki H, Ikeda A, Nagamine T, Mima T, Terada K, Nishitani

$\mathrm{N}$, et al. Cortical reflex negative myoclonus. Brain. 1994;

117 :477-486

Shibasaki H. Pathophysiology of negative myoclonus and asterixis. Adv Neurol. 1995; 67:199-209.

Shibasaki H. Physiology of negative myoclonus. Adv Neurol. 2002; 89:103-113

Stell R, Davis S, Carroll WM. Unilateral asterixis due to a lesion of the ventrolateral thalamus. J Neurol Neurosurg Psychiatry 1994; 57:878-880

Tassinari CA, Rubboli G, Shibasaki H. Neurophysiology of positive and negative myoclonus. Electroencephalogr Clin Neurophysiol. 1998; 107:181-195

Tatu L, Moulin T, Martin V, Monnier G, Rumbach L. Unilateral pure thalamic asterixis: clinical, electromyographic, and topographic patterns. Neurology. 2000; 54:2339-2342

Tijssen MA, Thom M, Ellison DW, Wilkins P, Barnes D, Thompson $\mathrm{PD}$, et al. Cortical myoclonus and cerebellar pathology.

Neurology. 2000; 54:1350-1356

Ugawa Y, Shimpo T, Mannen T. Physiological analysis of asterixis: silent period locked averaging. J Neurol Neurosurg Psychiatry. 1989; 52:89-93

Ugawa Y, Genba K, Shimpo T, Mannen T. Onset and offset of electromyographic (EMG) silence in asterixis. J Neurol Neurosurg Psychiatry. 1990; 53:260-262

Ugawa Y, Hanajima R, Terao Y, Kanazawa I. Exaggerated 16-20 $\mathrm{Hz}$ motor cortical oscillation in patients with positive or negative myoclonus. Clin Neurophysiol. 2003;114:1278-1284

Young RR, Shahani BT, Kjellberg RJ. Unilateral asterixis

produced by a discrete CNS lesion. Trans Am Neurol Assoc.

1976; 101:306-307

Young RR, Shahani BT. Asterixis: one type of negative myoclonus. Adv Neurol. 1986; 43:137-156 


\section{Figure legends}

Figure 1

Diffusion weighted MRI in two cases. Note high intensity signals (arrowhead) in the lateral part of the left thalamus in both cases. $\quad \mathrm{R}=$ right.

\section{Figure 2}

Surface EMG of the right extensor carpi radialis muscle showing two types of silent period (SP) in Case 2. In type $2 \mathrm{SP}$, note a sharp EMG transient dividing the SP into the first short SP and the second long SP.

Figure 3

Averaged EEG time-locked to the silent period (SP) onset in two cases. Note rhythmic activities in the left central area with a main positive peak of the oscillations coinciding with the onset of asterixis. Vertical line in each data corresponds to the onset of SP. Time scale is from $0.1 \mathrm{~s}$ before to $0.1 \mathrm{~s}$ after the SP onset. Number of sweeps for averaging was 49 for Case 1 and 127 for Case 2. 


\section{Figure 4}

Power change of EEG oscillations at 11 scalp electrodes, time-locked to the SP onset of the right hand in two cases. The magnified view of the data at electrode C3 for each case is shown on the right panel. Note an increase of power (shown in red) in beta band at the left central area, starting about $0.05 \mathrm{~s}$ before the SP onset. Blue segment of EMG data in RECR and RFCU (shown at the bottom for each case) corresponds to the asterixis. The color bar indicates relative change in the nature logarithm of power, at $95 \%$ confidence limits. For each electrode site, vertical axis $=$ frequency bands from 0 to $100 \mathrm{~Hz}$; horizontal axis $=$ time from $0.5 \mathrm{~s}$ before to $0.5 \mathrm{~s}$ after the $\mathrm{SP}$ onset (0). $\mathrm{ECR}=$ extensor carpi radialis; FCU = flexor carpi ulnaris; $\mathrm{L}=$ left; $\mathrm{R}=$ right; $\mathrm{EKG}$ $=$ electrocardiogram.

Figure 5

Waveforms of motor evoked potential (MEP) recorded from the first dorsal interosseous (FDI) muscles in response to transcranial magnetic stimulation (TMS) of the contralateral motor cortex (M1) in the two cases. Note the MEP amplitude was smaller on TMS of the affected (left: L) side compared with the unaffected (right: R) side. 
Figure 6

Connectivity of thalamic subregions to seven cortical areas calculated by probabilistic diffusion tractography. The affected lesion in the thalamus corresponds to the areas shown in light blue, green and purple, which were shown to be connected to M1, S1 and premotor cortex, respectively. Upper images show thalamic subregions on the axial, coronal and sagittal views, and lower images show the data on the enlarged axial view. The image on the left thalamus is shown in transmitted view in order to depict the lesion site. Low intensity region around crossbar indicates the position of infarction. Color code indicates connection to each cortical area as shown at the bottom of the figure. $\mathrm{R}=$ right; $\mathrm{L}=$ left $\mathrm{A}=$ anterior; $\mathrm{P}=$ posterior. 
Figure

Figure1

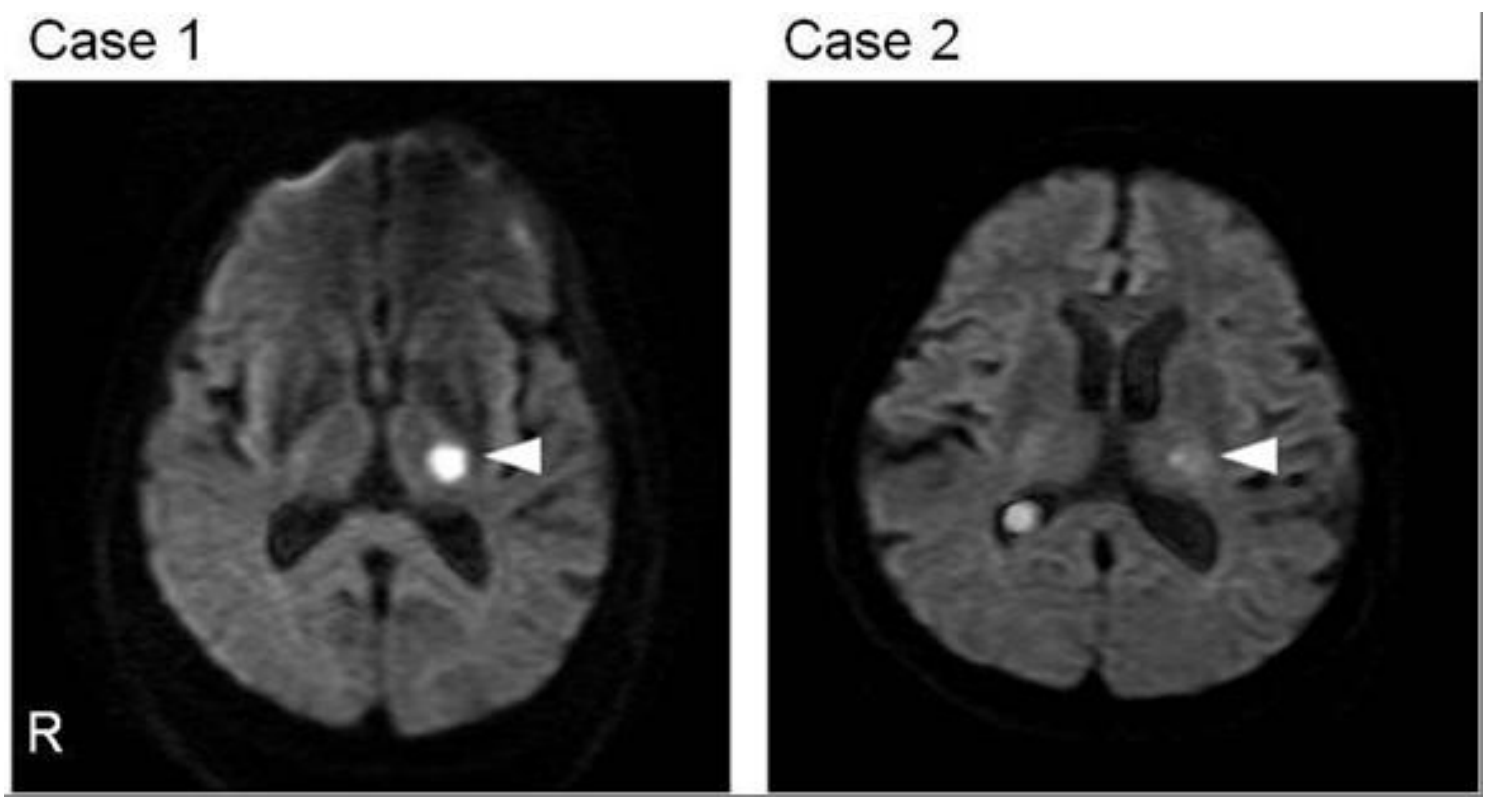

Figure2

\section{Type 1}
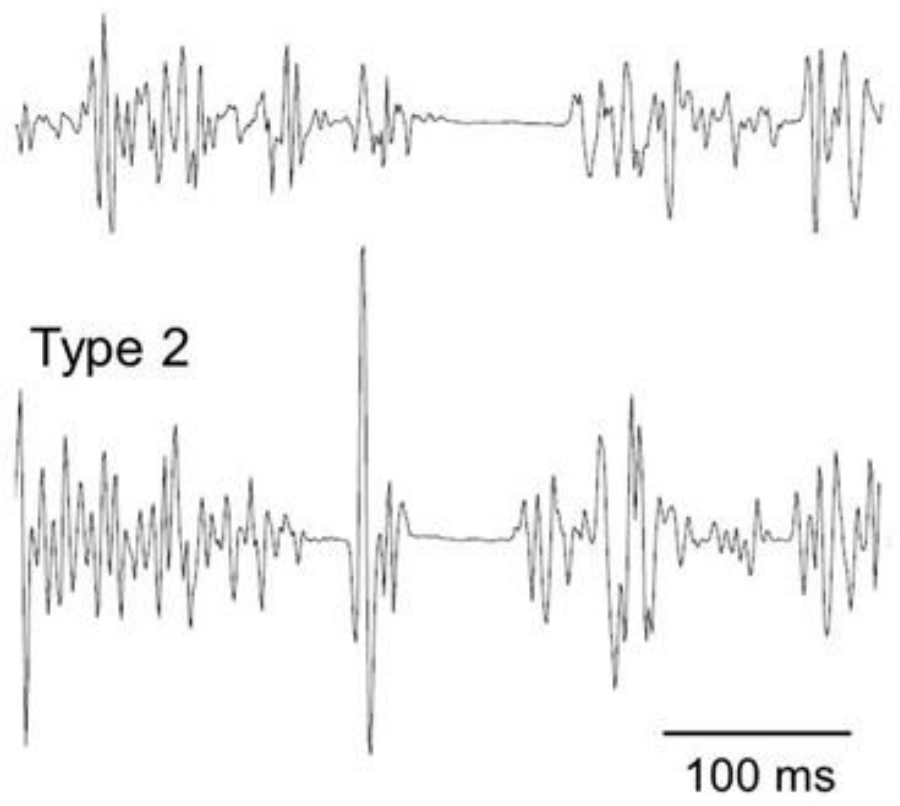
Figure3

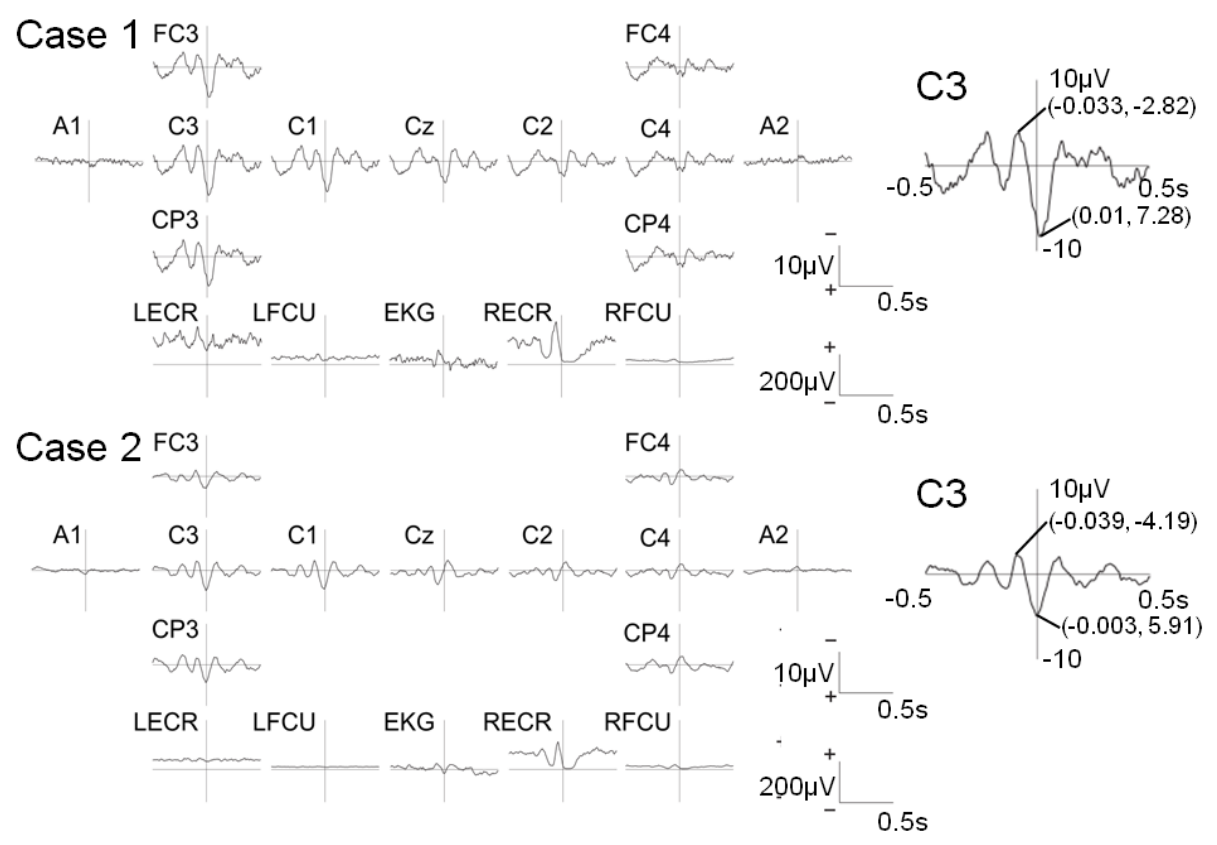

Figure4

Case 1 FC3

Case $2^{\mathrm{FC} 3}$
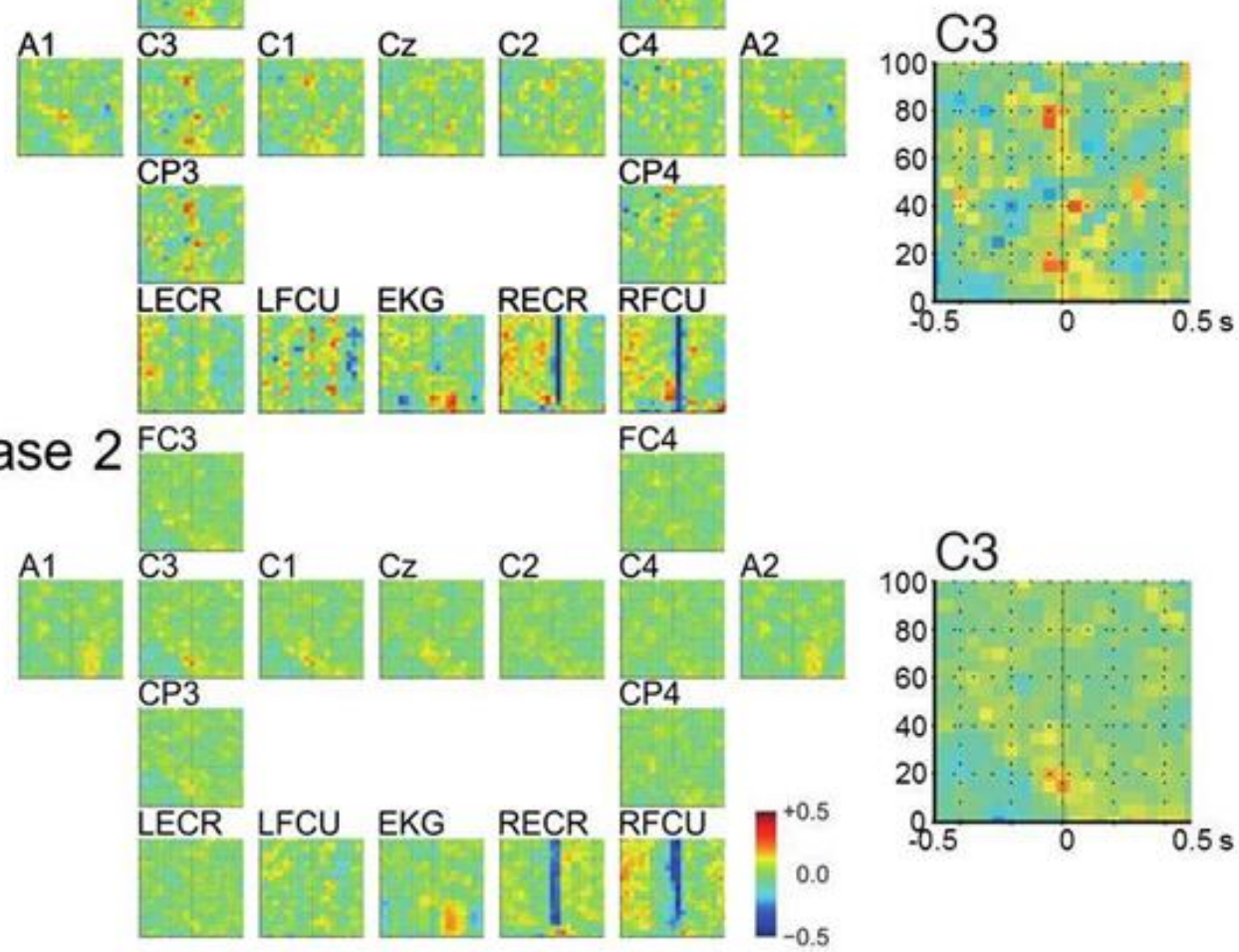
Figure5

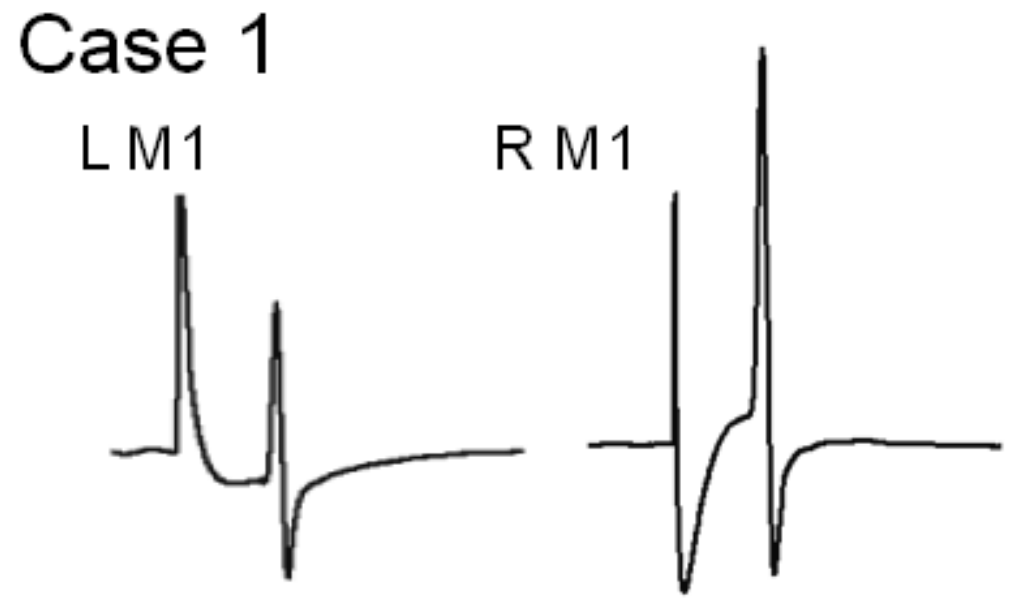

Case 2

L M1 R M1
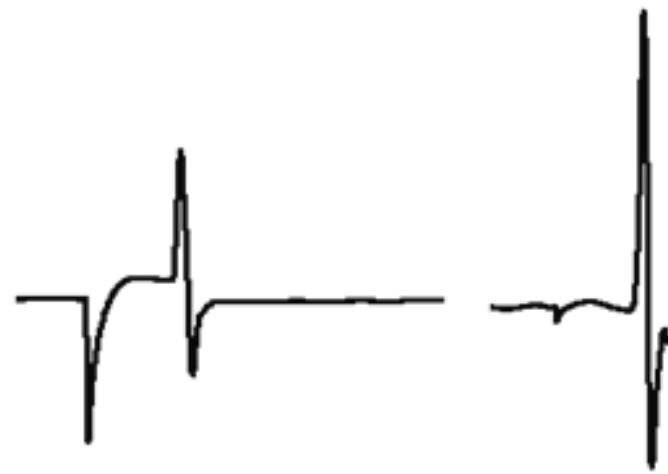

$1 \mathrm{mV}$

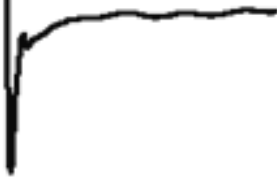

$40 \mathrm{~ms}$

Figure6 


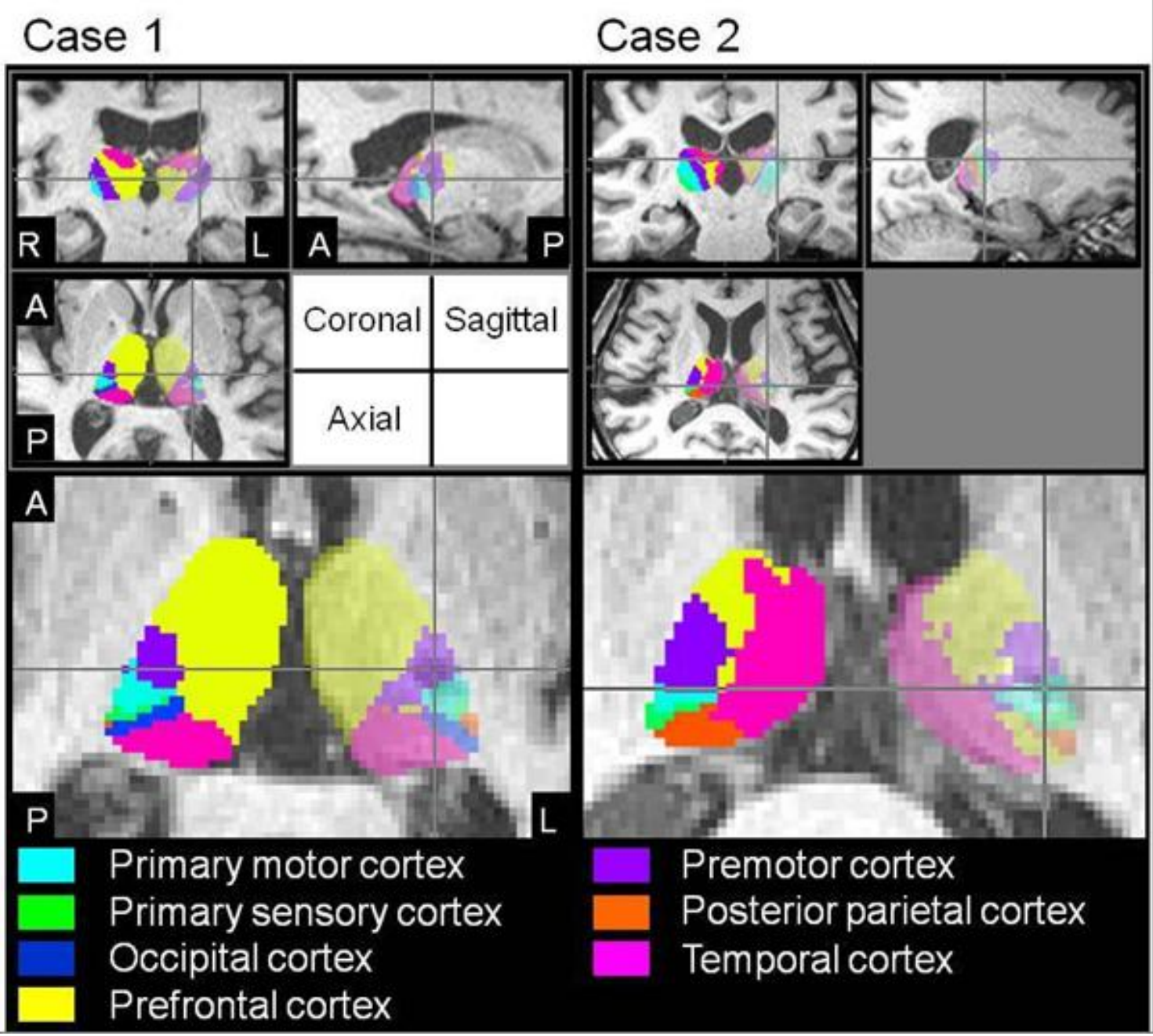

